\title{
SliClick - Em Direção à Criação de um Apresentador de Slides Vestível
}

\author{
Helder Yukio Okuno ${ }^{1}$, Gustavo Paiva Guedes ${ }^{1}$ \\ ${ }^{1}$ CEFET/RJ - Centro Federal de Educação Tecnológica Celso Suckow da Fonseca \\ Av. Maracanã, 229 - Rio de Janeiro - RJ - Brasil. \\ helder.okunodeic.cefet-rj.br, gustavo.guedes@cefet-rj.br
}

\begin{abstract}
Affective Computing has applications in the area of computer science to identify levels of anxiety through sensors and tools. This article presents the development and use of a wearable presentation device to detect the anxiety of individuals who perform in public. In addition, this device allows the speaker to move forward and backward slides. It also has a laser pointer and a vibracall engine, which notifies the speaker the near end of the presentation. Preliminary results indicate promising directions in the development of the wearable device, allowing users to assess their emotional states in their training for the talk.
\end{abstract}

Resumo. A Computação Afetiva possui aplicações na área da ciência da computação para identificar níveis de ansiedade através de sensores e ferramentas. Este artigo apresenta o desenvolvimento e uso de um apresentador de slides vestível para detectar a ansiedade de indivíduos que se apresentam em público. Além disso, este dispositivo vestível permite avançar e retroceder slides. Também possui um ponteiro laser e um motor de vibracall, que notifica o palestrante quando o fim da apresentação se aproxima. Resultados preliminares indicam direções promissoras no desenvolvimento do dispositivo vestível, permitindo usuários avaliarem seus estados emocionais em seus treinamentos para a palestra.

\section{Introdução}

A Computação Afetiva (CA) é um dos tópicos de pesquisa mais ativos atualmente, devido ao seu amplo espectro de aplicação, que diz respeito a conhecimentos multidisciplinares como a psicologia, cognição, fisiologia e a ciência da computação [Tao and Tan 2005]. No âmbito da computação, podemos destacar as pesquisas na área de Inteligência Artificial, que se encontra inserida nesse contexto para proporcionar que os computadores disponham de capacidade de observação, interpretação e geração de características de afeto [Picard 1995] para, dessa maneira, aperfeiçoar a interação homem-máquina.

As linhas de pesquisa mais amplas da CA estão relacionadas à detecção de emoções e à indução e simulação das emoções [Picard 1995]. A detecção de emoções pode ser realizada a partir da análise dos batimentos cardíacos, gestos, expressões faciais, ou a partir de dispositivos vestíveis dotados de sensores e ferramentas que permitem o reconhecimento de padrões afetivos do usuário [Picard and Healey 1997]. Na indução de emoções, alguns estudos envolvem a apresentação filmes aos indivíduos com 
VI Congresso Brasileiro de Informática na Educação (CBIE 2017)

Anais dos Workshops do VI Congresso Brasileiro de Informática na Educação (WCBIE 2017)

o propósito de analisar o entusiasmado [Picard et al. 2001]. Já na área de simulação de emoções, destacamos os estudos baseados na teoria da abordagem cognitiva da emoção [Egges et al. 2004].

O contexto da detecção de emoções, foco deste trabalho, pode ter diversas aplicações, como a área da segurança, identificando textos de potenciais predadores sexuais [Rodrigues et al. 2017]; a área automobilística, com carros inteligentes que identificam o déficit de atenção do motorista [Clark et al. 2016]; a área da saúde, nas pesquisas envolvendo a mente de indivíduos autistas [El Kaliouby et al. 2006]; a área da computação, detectando a ansiedade por meio de sensores em dispositivos vestíveis [Rani et al. 2007].

A ansiedade acomete indivíduos no mundo todo [Pull 2008, Michael et al. 2007] e pode ser classificada de duas formas: a primeira, conhecida por ansiedade cognitiva, em que ocorrem pensamentos negativos ou duvidosos; a segunda é denominada ansiedade somática, em que ocorre uma elevação na frequência cardíaca e aumento na quantidade de suor nas mãos [Borkovec and Inz 1990]. A ansiedade pode ser caracterizada ainda como um sentimento bom ou ruim [Gray 1972]. Por exemplo, o estado emocional ansioso antes do nascimento de um filho pode ser apontado como um estado bom. Por outro lado, o estado emocional ansioso antes de se iniciar uma apresentação em público pode ser considerado ruim [Muniz et al. 2016].

A ansiedade em se apresentar em público independe de gênero, etnia e idade [Phillips et al. 1997] e é bastante frequente em palestrantes [Pull 2012]. Os sintomas associados à ansiedade em falar em público são diversos, dentre eles são destacados a falta de ar, suor em excesso, desconforto digestivo ou o esquecimento de algo que se conhece ou estava prestes a dizer [Katz 2000]. Esta modalidade de apresentação é bastante usual em trabalhos acadêmicos (e.g., seminários) e diversos estudantes relatam sentir o aumento da ansiedade durante essas apresentações [Zeggio Perez Figueredo and Vieira Barbosa 2008].

O tempo de apresentação é um outro elemento que pode trazer dificuldades na realização de uma palestra. O palestrante, muitas vezes, necessita expor o conteúdo programado dentro de um espaço de tempo limitado, entretanto ocorrem variações na velocidade da fala, o que pode resultar no término precoce ou após o tempo máximo permitido [Gregersen 2009]. Recursos tecnológicos (e.g., lasers) podem impulsionar ainda mais as dificuldades do apresentador, resultando, por exemplo, em tremores nas mãos ao manusear o dispositivo [Krantz 2008].

Sabe-se ainda que o palestrante necessita utilizar, muitas vezes, o microfone e o passador de slides com laser, fazendo com que suas duas mãos estejam ocupadas. No entanto, diversos estudos destacam que os humanos utilizam as duas mãos (por meio dos gestos) como parte integrante da comunicação e da linguagem, completando a mensagem verbal percebida por parte da audiência [McNeill 1992, Jaimes and Sebe 2007].

A partir das perspectivas apresentadas, destacamos que o presente trabalho tem o objetivo de contribuir com o desenvolvimento de um dispositivo vestível, em inglês wearable device, denominado SliClick. O SliClick consiste em um apresentador de slides, com as funções de avançar e retroceder slides, laser integrado e um temporizador configurável, que informa por meio de um sinal vibratório a proximidade do término do tempo 
VI Congresso Brasileiro de Informática na Educação (CBIE 2017)

Anais dos Workshops do VI Congresso Brasileiro de Informática na Educação (WCBIE 2017)

da apresentação. Os componentes utilizados para a construção do dispositivo vestível foram: microcontroladores Arduino, placas de radiofrequência (RF), diodo laser e o motor vibracall.

Além disso, o SliClick possui um sensor de resposta galvânica de pele (GSR) que mede a atividade elétrica das glândulas sudoríparas que produzem suor nas pontas dos dedos para identificar emoções e situações que causem ansiedade. Dessa maneira, espera-se que os usuários do SliClick possam avaliar seus estados emocionais em seus treinamentos para palestras, identificando e lidando com suas fraquezas. Vale ressaltar que dispositivos vestíveis que reconhecem estados afetivos são denominados dispositivos afetivos vestíveis [Picard and Healey 1997] ou, em inglês, affective wearables.

Este trabalho é dividido em mais 4 seções. Na Seção 2 são apresentados os trabalhos relacionados a esta pesquisa. A Seção 3 descreve a ferramenta proposta. Os resultados preliminares são descritos na Seção 4. Por fim, na Seção 5, são discutidas as conclusões e trabalhos futuros.

\section{Trabalhos relacionados}

Várias abordagens foram propostas na literatura para detectar ou tratar a ansiedade por meio da tecnologia [Miranda et al. 2014]. Algumas dessas propostas abordam o uso de dispositivos wearable e outras destacam a importância do uso de sensores vestíveis para monitoramento e tratamento de doenças crônicas, distúrbios neurológicos e problemas de saúde mental, como o diabetes, transtorno do espectro autista, depressão e transtornos da ansiedade [Fletcher et al. 2010]. Também podem ser destacados os trabalhos desenvolvidos com realidade virtual para a compreensão, avaliação e tratamento de problemas de saúde mental ao longo dos últimos 15 anos [Riva 2005].

O trabalho proposto em [Repetto et al. 2013] utiliza a realidade virtual para que os indivíduos participem de uma terapia de exposição e analisa suas frequências cardíacas. Os resultados foram mensurados com base nas pontuações do questionário de psicometria proposto pelos autores, realizados no pré e pós tratamento. No questionário de psicometria aplicado antes do tratamento, o grupo de indivíduos obteve a média de 26,65 pontos. Após o tratamento, um novo questionário foi aplicado a média obtida foi de 14, 50 pontos, o que representa um decréscimo de $45,59 \%$.

Em [Rennert and Karapanos 2013], os autores propõem a utilização de uma câmera para capturar o áudio e o vídeo dos pacientes. Além disso, os autores capturam a frequência cardíaca e a posição geográfica desses paciente. Esses dados são enviados a uma interface web para identificar o momento em que o paciente obteve uma alta frequência cardíaca e criar relações com os fatos externos capturados através da câmera. O usuário ainda podem apagar registros que são falsos-positivos, como uma frequência cardíaca alta ao realizar uma atividade física. Os autores destacam que esse grande volume de dados gerados diariamente pode ajudar ao especialista a entender melhor os transtornos do paciente.

Destacamos também os sistemas capazes de identificar o nível de ansiedade por meio de textos e aplicam a cromoterapia para mudar a cor da tela a fim de diminuir o nível de ansiedade do indivíduo [Muniz et al. 2016]. Nesse trabalho, são utilizados as cores vermelha e azul, em a primeira cor está associada à tendência de excitação e a 
VI Congresso Brasileiro de Informática na Educação (CBIE 2017)

Anais dos Workshops do VI Congresso Brasileiro de Informática na Educação (WCBIE 2017)

segunda ao relaxamento. Conforme o indivíduo escreve o texto na ferramenta, palavras são pesquisadas em um dicionário afetivo - uma ferramenta para análise de textos que categoriza palavras e identifica traços de ansiedade, felicidade, tristeza, dentre outros.

Podemos encontrar na literatura diversos trabalhos voltados ao tratamento e acompanhamento de indivíduos com transtornos e doenças crônicas utilizando dispositivos vestíveis [Ćosić et al. 2010]. Entretanto, não foram encontrados trabalhos com o foco na ansiedade normal, temporária e autolimitada, somente em ansiedade patológica. É nesse aspecto que o presente trabalho apresenta sua contribuição: um apresentador de slides no formato de uma luva vestível que auxilia indivíduos em palestras e apresentações em público.

\section{Sliclick}

Nesta seção descrevemos o SliClick, um apresentador de slides no formato de uma luva para auxiliar indivíduos em apresentações públicas que utilizem slides. Um palestrante que necessitava portar um microfone em uma das mãos e na outra, um apresentador de slides com laser, foi a inspiração para o desenvolvimento deste trabalho. Ao longo de sua apresentação, o palestrante gesticulava para exemplificar ou explicar um assunto, assim, guardava o apresentador de slides no bolso. Ao retirar o apresentador do bolso, alguns botões eram apertados erroneamente, voltando slides já apresentados ou avançando sua apresentação. Foi identificado também o aspecto do tempo de apresentação, pois houve um avanço de mais de 20 minutos além dos 50 minutos limitados à apresentação.

O desenvolvimento do SliClick ocorreu em três módulos. O primeiro módulo, ilustrado na Figura 1, representa a luva desenvolvida. É composto por um microcontrolador Arduino, placa de radiofrequência, diodo laser, motor de vibracall, sensor GSR e 3 baterias do tipo botão, interligadas em série, totalizando 9 volts.

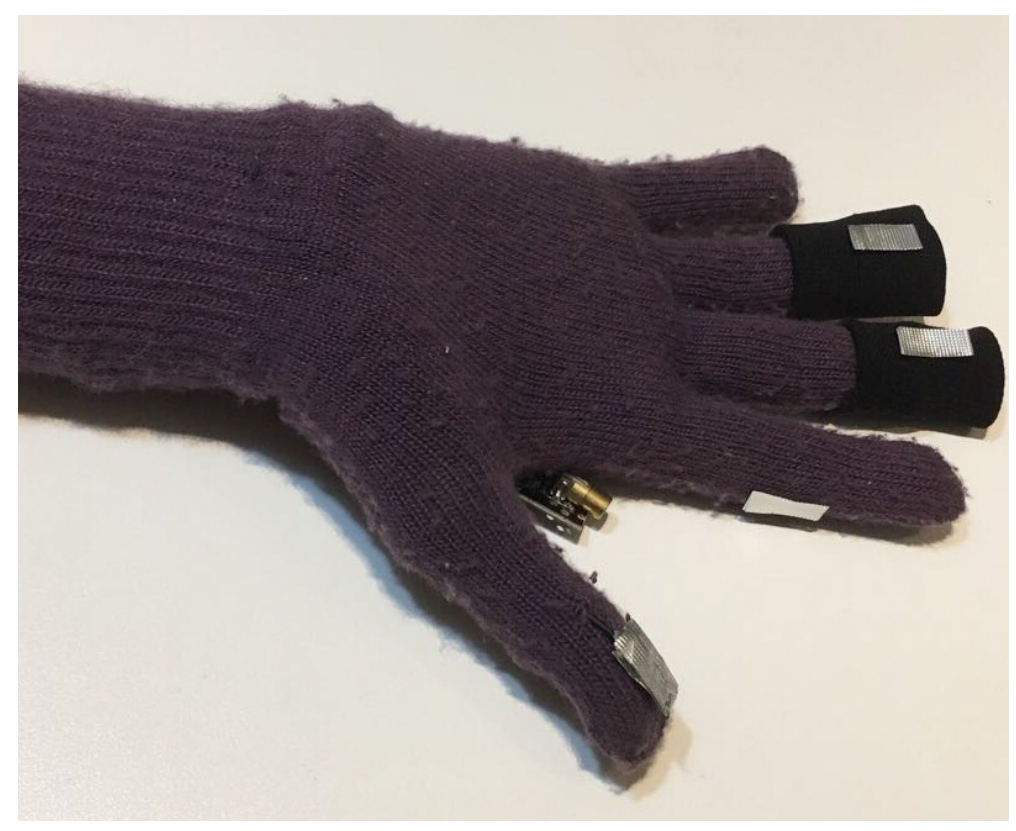

Figura 1. Protótipo da luva SliClick: são apresentados 4 contatos secos (dedo polegar, indicador, médio e anelar), o diodo laser na parte lateral do dedo indicador e os sensores GSR. 
VI Congresso Brasileiro de Informática na Educação (CBIE 2017)

Anais dos Workshops do VI Congresso Brasileiro de Informática na Educação (WCBIE 2017)

São apresentados também 4 contatos secos instalados no dedo polegar, indicador, médio e anelar. Ao realizar o contato do dedo polegar com o indicador, o diodo laser é ativado, permitindo ao apresentador apontar algo em seu slide, evidenciando a atenção da audiência. O contato do dedo polegar com o dedo médio avança o slide e para retornar ao slide anterior, basta que o palestrante realize o contato do dedo polegar com o anelar. Os sensores GSR estão presentes nos dedos indicador e anelar, permitindo-se a detecção dos níveis de ansiedade para o treinamento e nas apresentações em público.

A Figura 2 ilustra o esquema de ligação dos componentes instalados fisicamente na luva. A letra A representando o microcontrolador Arduino Nano. Optamos pelo modelo Nano por ser compacto e de fácil acomodação na luva. A letra B ilustra o compartimento de baterias do tipo botão, ligadas em série, para gerar a voltagem necessária e alimentar todos os demais componentes conectados ao microcontrolador. Utilizamos um transmissor e um receptor de RF de $433 \mathrm{Mhz}$, respectivamente representados com a letra C e D no diagrama, possibilitando a comunicação sem fio entre a luva e o computador do palestrante. As letras E, F e G representam os contatos secos para avançar o slide, retornar slides anteriores e habilitar o diodo laser, respectivamente. O componente do diodo laser é caracterizado com a letra $\mathrm{H}$. Por fim, a letra I representa o motor vibracall, para indicar ao palestrante que os últimos minutos de seu tempo estão se aproximando.

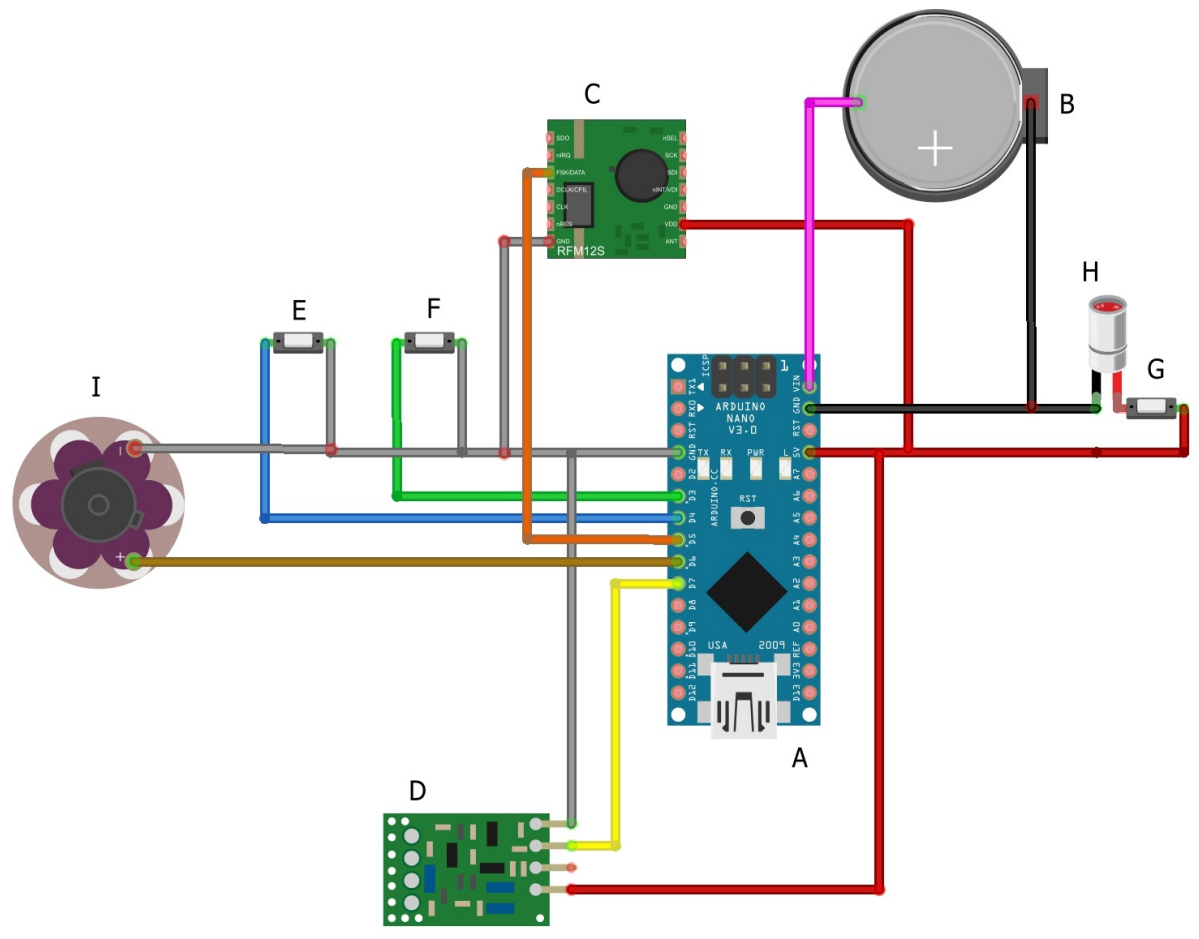

Figura 2. Esquema de ligações do protótipo da luva com os respectivos componentes.

O segundo módulo, que realiza a comunicação da luva com o computador do palestrante, foi desenvolvido com um outro microcontrolador Arduino Nano, conectado à porta USB do computador. Na Figura 3 ilustramos as conexões desse segundo Arduino, representado pela letra $\mathrm{J}$; as letras $\mathrm{K}$ e L ilustram, respectivamente, o transmissor e o receptor de RF, que operam em 433Mhz; a letra M simboliza o computador do palestrante, 
VI Congresso Brasileiro de Informática na Educação (CBIE 2017)

Anais dos Workshops do VI Congresso Brasileiro de Informática na Educação (WCBIE 2017)

que deverá estar conectada ao Arduino (letra J) via USB.

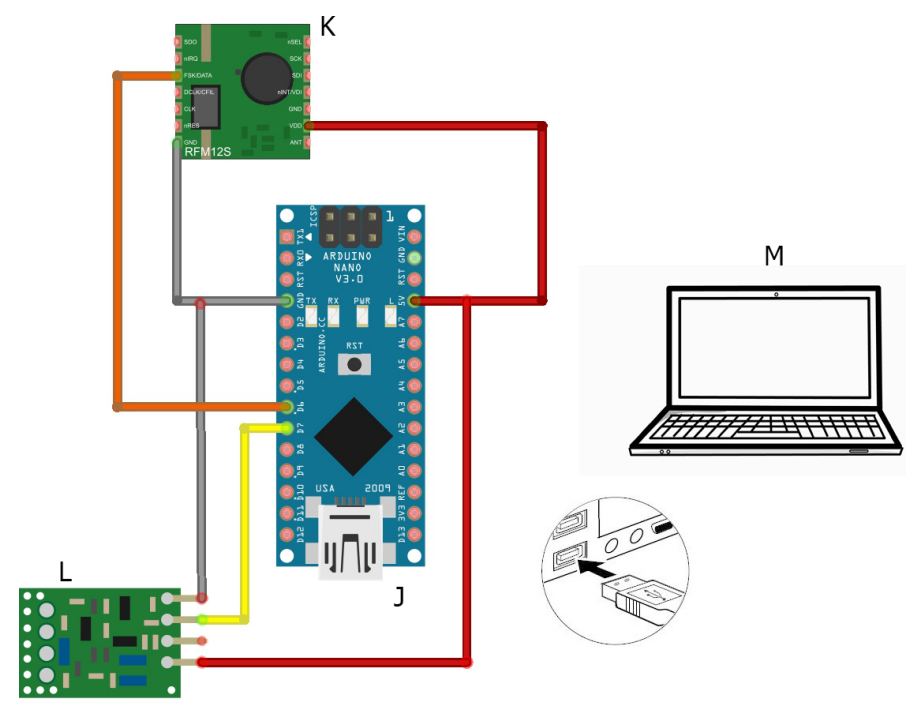

Figura 3. Esquema de ligações do protótipo conectado ao computador do palestrante.

O terceiro módulo, representado na Figura 4 e denominado servidor, foi codificado em linguagem Java. Esse aplicativo comunica-se pela porta USB do computador do palestrante com o microcontrolador Arduino representado pela letra J na Figura 3. Em seguida, esse microcontrolador se comunica com o microcontrolador da luva (representado pela letra A na Figura 2) utilizando as placas de RF.

Em momento anterior à sua apresentação, o palestrante necessita configurar o aplicativo do servidor, inserindo o tempo total da apresentação e quantos minutos antes do término desse tempo, o palestrante deseja ser notificado pelo motor de vibracall. Ao final dessa configuração, o palestrante deve clicar em iniciar no aplicativo que irá atribuir o horário inicial e no momento agendado, enviará a notificação de vibração para a luva, por meio da comunicação RF.

\section{Resultados preliminares}

Nesta seção descrevemos os resultados preliminares encontrados durante o desenvolvimento do SliClick. Os experimentos foram realizados com o objetivo de avaliar o funcionamento do sistema e a comunicação entre os componentes. Dessa maneira, foram divididos em quatro fases.

Na primeira fase, testamos o avanço e retrocesso dos slides utilizando os sensores de RF (conforme mencionado na Seção 3, fechando o contato do polegar com os dedos indicador e anelar). Essa fase do experimento foi executada com bastante sucesso, sendo possível avançar e retroceder os slides de modo correto.

$\mathrm{Na}$ segunda fase, produzimos os testes para habilitar/desabilitar o apontador a laser. Essa fase também foi executada com sucesso. O laser permaneceu ligado enquanto havia contato e desligado conforme o contato fosse desfeito.

$\mathrm{Na}$ terceira fase, testamos o aplicativo para configurar o aviso ao palestrante antes do término da apresentação. Configuramos a duração da apresentação com 15 minutos e 


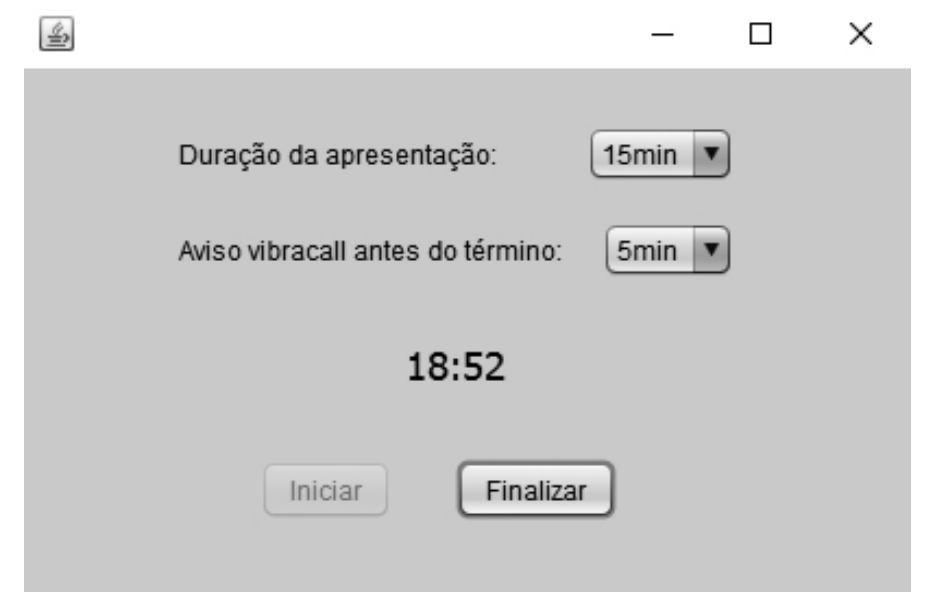

Figura 4. Protótipo do aplicativo Sliclick instalado no computador do palestrante. É possível configurar o tempo de duração e o tempo para o envio do aviso antes do término da apresentação.

o aviso para 5 minutos antes do final. $\mathrm{O}$ aviso foi enviado com sucesso, ou seja, faltando 5 minutos para o fim a apresentação.

Na quarta e última fase, testamos o sensor de resposta galvânica da pele, sendo possível observar, em tempo real, as alterações nos valores. Nessa fase do projeto, esses valores foram apenas exibidos no console da aplicação.

\section{Conclusões preliminares e trabalhos futuros}

A Computação Afetiva é uma áreas de pesquisa bastante atual. Possui uma linha de pesquisa relacionada à detecção de emoções, que abrange projetos em diferentes áreas de pesquisa, dentre elas, a ciência da computação. Nessa área, podemos destacar as aplicações capazes de detectar a ansiedade a partir de sensores, por exemplo, o sensor de resposta galvânica da pele.

Nesse cenário, destacamos que a ansiedade de falar/apresentar em público é uma condição bastante comum, levando a tremores, náusea, confusão ao avançar/retroceder os slides e dificuldade de finalizar a apresentação no tempo correto. Esses aspectos também foram apresentados no contexto da educação, em que muitos alunos relatam ansiedade intensa durante apresentações de seminários. Além disso, destacamos que, muitas vezes, o palestrante sente necessidade de gesticular com as duas mãos.

Com isso, foi proposto um dispositivo vestível de apresentação capaz de detectar ansiedade. Esse dispositivo, permite avançar e retroceder slides, capturar a condutância da pele com um GSR, ligar/desligar um laser embutido, além de avisar com um sensor vibracall quando a apresentação está próxima do fim.

Os resultados preliminares indicaram o potencial do protótipo. A comunicação entre os componentes foi realizada com sucesso. Como próximos trabalhos, realizaremos os experimentos com os componentes em conjunto e em apresentações reais. Também pretendemos realizar experimentos capazes de detectar os momentos de maior ansiedade nas apresentações. 
VI Congresso Brasileiro de Informática na Educação (CBIE 2017)

Anais dos Workshops do VI Congresso Brasileiro de Informática na Educação (WCBIE 2017)

\section{Referências}

Borkovec, T. and Inz, J. (1990). The nature of worry in generalized anxiety disorder: A predominance of thought activity. Behaviour research and therapy, 28(2):153-158.

Clark, H. E. et al. (2016). Limited self-driving vehicle automation: Age differences in the takeover of vehicle control, engagement in non-driving-related activities and opinions of the technology.

Ćosić, K., Popović, S., Kukolja, D., Horvat, M., and Dropuljić, B. (2010). Physiologydriven adaptive virtual reality stimulation for prevention and treatment of stress related disorders. CyberPsychology, Behavior, and Social Networking, 13(1):73-78.

Egges, A., Kshirsagar, S., and Magnenat-Thalmann, N. (2004). Generic personality and emotion simulation for conversational agents. Computer animation and virtual worlds, 15(1):1-13.

El Kaliouby, R., Picard, R., and BARON-COHEN, S. (2006). Affective computing and autism. Annals of the New York Academy of Sciences, 1093(1):228-248.

Fletcher, R. R., Poh, M.-Z., and Eydgahi, H. (2010). Wearable sensors: opportunities and challenges for low-cost health care. In Engineering in Medicine and Biology Society (EMBC), 2010 Annual International Conference of the IEEE, pages 1763-1766. IEEE.

Gray, J. A. (1972). The structure of the emotions and the limbic system. Physiology, Emotions and Psychosomatic Illness, pages 87-129.

Gregersen, T. (2009). Recognizing visual and auditory cues in the detection of foreignlanguage anxiety. TESL Canada Journal, 26(2):46-64.

Jaimes, A. and Sebe, N. (2007). Multimodal human-computer interaction: A survey. Computer vision and image understanding, 108(1):116-134.

Katz, L. (2000). Public speaking anxiety. UTM Counseling and Career Services, 1:1-3.

Krantz, W. (2008). Ac 2008-74: Oral communication skills workshop for students in engineering and applied science. age, 13:1.

McNeill, D. (1992). Hand and mind: What gestures reveal about thought. University of Chicago press.

Michael, T., Zetsche, U., and Margraf, J. (2007). Epidemiology of anxiety disorders. Psychiatry, 6(4):136-142.

Miranda, D., Calderón, M., and Favela, J. (2014). Anxiety detection using wearable monitoring. In Proceedings of the 5th Mexican Conference on Human-Computer Interaction, page 34. ACM.

Muniz, R. d. S., Rodrigues, R. G., and Guedes, G. P. (2016). Croca - cromoterapia e computação afetiva: auxiliando os estados de ansiedade. In Webmedia '16 Proceedings of the 22nd Brazilian Symposium on Multimedia and the Web, volume 2, pages 145148.

Phillips, G. C., Jones, G. E., Rieger, E. J., and Snell, J. B. (1997). Normative data for the personal report of confidence as a speaker. Journal of Anxiety Disorders, 11(2):215 220 . 
VI Congresso Brasileiro de Informática na Educação (CBIE 2017)

Anais dos Workshops do VI Congresso Brasileiro de Informática na Educação (WCBIE 2017)

Picard, R. W. (1995). Affective Computing. MIT Press, Cambridge, MA, USA.

Picard, R. W. and Healey, J. (1997). Affective wearables. In Wearable Computers, 1997. Digest of Papers., First International Symposium on, pages 90-97. IEEE.

Picard, R. W., Vyzas, E., and Healey, J. (2001). Toward machine emotional intelligence: Analysis of affective physiological state. IEEE transactions on pattern analysis and machine intelligence, 23(10):1175-1191.

Pull, C. B. (2008). Recent trends in the study of specific phobias. Current opinion in psychiatry, 21(1):43-50.

Pull, C. B. (2012). Current status of knowledge on public-speaking anxiety. Current opinion in psychiatry, 25(1):32-38.

Rani, P., Sarkar, N., and Adams, J. (2007). Anxiety-based affective communication for implicit human-machine interaction. Advanced Engineering Informatics, 21(3):323334.

Rennert, K. and Karapanos, E. (2013). Faceit: Supporting reflection upon social anxiety events with lifelogging. In CHI'13 Extended Abstracts on Human Factors in Computing Systems, CHI EA '13, pages 457-462, New York, NY, USA. ACM.

Repetto, C., Gaggioli, A., Pallavicini, F., Cipresso, P., Raspelli, S., and Riva, G. (2013). Virtual reality and mobile phones in the treatment of generalized anxiety disorders: A phase-2 clinical trial. Personal Ubiquitous Comput., 17(2):253-260.

Riva, G. (2005). Virtual reality in psychotherapy. Cyberpsychology \& behavior, 8(3):220230.

Rodrigues, R. G., Pereira, W. W., Bezerra, E., and Guedes, G. P. (2017). Inferência de idade utilizando o LIWC: identificando potenciais predadores sexuais. In XXXVII Congresso da Sociedade Brasileira de Computação.

Tao, J. and Tan, T. (2005). Affective computing: A review. pages 981-995. In International Conference on Affective Computing and Intelligent Interaction. Springer-Verlag Berlin Heidelberg.

Zeggio Perez Figueredo, L. and Vieira Barbosa, R. (2008). Fobia social em estudantes universitários. ConScientiae Saúde, 7(1). 